\title{
Mechanochemical Methods in the Production of High Purity Gases
}

\author{
Konstantin Chuntonov ${ }^{1 *}$, Boris Verbitsky ${ }^{1}$, Alexey O. Ivanov ${ }^{2}$, Victor L. Kozhevnikov ${ }^{3}$ \\ ${ }^{1}$ Mechemlab Ltd, Nesher, Israel \\ ${ }^{2}$ Institute of Mathematics and Computer Sciences, Ural Federal University, Yekaterinburg, Russia \\ ${ }^{3}$ Institute of Solid State Chemistry, Russian Academy of Sciences, Ural Branch, Yekaterinburg, Russia \\ Email: *konstantin@mechemlab.com
}

How to cite this paper: Chuntonov, K. Verbitsky, B., Ivanov, A.O. and Kozhevnikov, V.L. (2018) Mechanochemical Methods in the Production of High Purity Gases. Materials Sciences and Applications, 9, 489-501.

https://doi.org/10.4236/msa.2018.95034

Received: March 30, 2018

Accepted: May 20, 2018

Published: May 24, 2018

Copyright (C) 2018 by authors and Scientific Research Publishing Inc. This work is licensed under the Creative Commons Attribution International License (CC BY 4.0).

http://creativecommons.org/licenses/by/4.0/

\begin{abstract}
The development of advanced and cost-effective methods is of prime importance for manufacturers of high purity gases. In this paper a new strategy in the development of gas flow purification technologies is described, where instead of adsorbents reactants are used, in which not only the surface is used in gas capturing but the entire volume of the material. Moreover, the reactants are activated in the gas flow by a controlled mechanical tool, which keeps the sorption kinetics at the required highest level and reduces the unproductive losses of the consumed reactant almost to zero. The advantages of the method are demonstrated with the examples of two novel gas purification units that are distinguished with uncomplicated design, serviceability and ultimately high purification efficiency.
\end{abstract}

\section{Keywords}

High Purity Gases, Chemisorption, Reactants, Mechanical Activation, Gas Purification

\section{The Problem of Gas Purification Efficiency}

In many fields like microelectronics, medicine, semiconductor production, pharmacology, welding, scientific research, etc. there is a need in high purity gases. At the same time the production of high- and ultra-pure gases is a multistage and very expensive process. For this reason, the search is currently rather important for efficient and resource-saving gas purification technologies.

One of the most common methods of gas purification is to pass the gas through a porous medium that can capture the undesirable admixtures by forming strong chemical bonds. Gas purifiers that work according to this prin- 
ciple may utilize metallic chemisorbents in the form of a porous body or powder [1] [2] [3] [4] [5]. The best gas purity grade can be attained when powder alloys of highly active metals (Me) of I and II groups are used for capturing gas impurities via respective chemical reactions that involve the entire volume of reactant particles [6]. At normal temperatures, which for gas purifiers are typically their operational temperatures, reactants greatly surpass traditional getters on the basis of transition metals [5] [7] [8]. However, it is found also in works [5] [7] [8] that the consumption $(\eta)$ of the reactant, i.e. the mass fraction of the material that has reacted with the gas, is rather small, $0.1 \leq \eta \leq 0.2$, in such sorbers with fixed bed. Therefore, there is a potential to increase the efficiency of the reactant using by 5 - 10 times, and this is the starting point for the present work.

Two questions arise here. First, why the reactants, which are able to completely react with impurity gases at room temperature, cannot attain their full sorption capacity; and second, how the gas purification process should be organized in order for the reactant to achieve its sorption maximum.

The impurity distribution in the gas flow through the powder charge of the tubular gas purifier may help to clarify the first question. The distribution pattern obtained in work [9] is shown in Figure 1. It is represented by the family of curves for impurity concentration $u\left(\xi, \tau_{i}\right)$, where $u$ is the concentration of gas impurity, $\xi$ is the longitudinal coordinate, and $\tau_{i}$ is the discrete values of time forming the sequence $\tau_{1}<\tau_{2}<\tau_{3}<\tau_{c}$ The gas purity is seen do decrease with time, and the process is to be halted at the time moment $\tau_{c}$ when the impurity content in the end product has attained maximum allowable level $u_{c}$

By that moment the size of the powder zone consisting only of completely exhausted particles is measured by the length of the region $\left[0, \xi_{c}\right]$, which is the shorter the higher the requirements to the purity of the gas product. However, the shorter the region $\left[0, \xi_{c}\right]$ the larger the share of the workable powder, which at this technology gets into the production waste. Losses of the reactant, as it was mentioned earlier, are $80 \%-90 \%$ of the initial mass and this is the price, which has to be paid today for the production of high purity and ultra pure gases.

The reason of these losses is an abrupt decrease of the rates of capturing gases from the moment when the volume of the powder particles is involved in the purification process. The rate of capturing the impurity by the elementary layer $d \xi$ of the powder charge from this moment becomes slower than the rate of the impurity coming into the given layer. As the result the distribution $u(\xi, \tau)$ shown in Figure 1 appears, which is responsible for the passive behavior of the powder reactant in flow gas purification columns. That is, in the case of reactants the problem of raising the efficiency of the purification process comes down to the problem of accelerating chemical reactions in the system gas/powder.

Several approaches can be used to solve the problem including the using of nano-sized reactants [3] [10] [11], heating [12] [13] [14], and mechanical activation [15]. The first two methods do not result in any sufficient improvement of the efficiency although accompanying costs considerably increase. In the present 


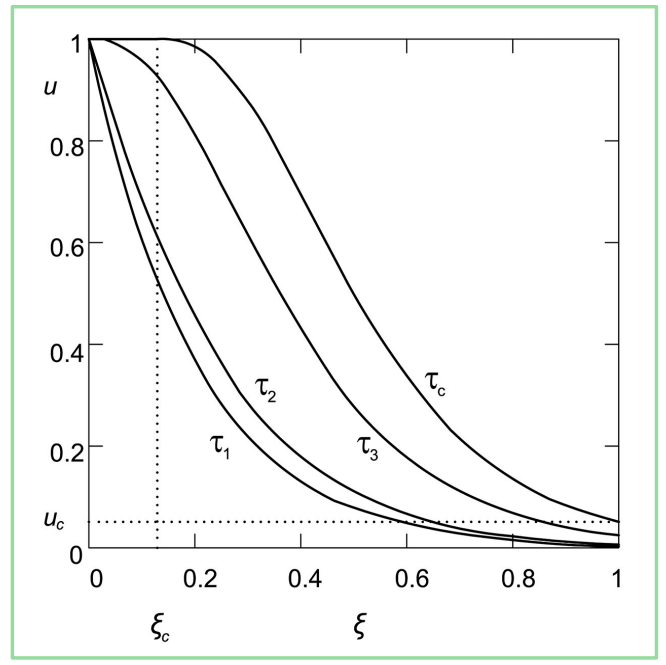

Figure 1. The distribution of impurity concentration in the gas phase along the sorption column at different moments of time. Here, $u$ is dimensionless impurity concentration, $\xi$ is dimensionless longitudinal coordinate, $\tau$ is dimensionless time, $\xi_{c}$ is the boundary of the exhausted powder, and $u_{c}$ stands for the maximal allowed impurity concentration in the outlet gas.

work we use the mechanochemical variant of purification when the reactant is activated in gas medium with the aid of mechanical forces. Let us also mention that both the local heating of the sorption material and the elements of nanotechnologies are present in mechanochemical processes as their integral part.

The novel mechanochemical apparatus [16] and the reactive sorber (patent pending) are described below. The units provide increased rate of impurities capturing and minimized loss of the purifying material.

\section{Mechanochemical Sorption Apparatus}

The working principle of the apparatus is shown in Figure 2.

Gas $\mathrm{X}$ with impurity $\mathrm{Y}$ is fed to column 1 along line 5 through filter 4 and passes through the purification powder, which is coming from disintegrator 2. The powder is produced by milling a reactive ingot with the aid of a mechanical tool connected with the outside actuator 3 via a feed through. Impurity Y reacts with Me particles in a thin layer of fresh powder 11 forming a solid product MeY and gas $\mathrm{X}$ leaves the column through filter 9. Particles $\mathrm{MeY}$ are gradually collected in the bottom part of the column as waste 10 while the powder pile grows upwards due to the poring particles 6 .

The respective bench prototype in Figure 3 is equipped with motionless Me ingot holder while the milling cutter performs translational and rotational movements.

Another model in Figure 4 is based on combination of linear movements of the milling cutter with rotation of the ingot holder. 


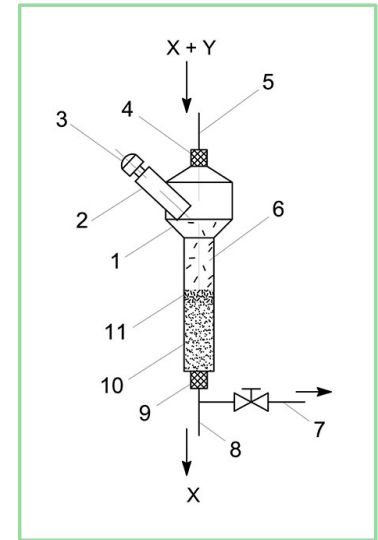

Figure 2. The sketch of mechanochemical sorption apparatus; 1 -sorption column, 2-disintegrator, 3-actuator, 4-filter, 5-inlet pipe, 6-Me particles, 7-sampling line, 8-gas outlet, 9-filter, 10-pile of waste $\mathrm{MeY}, 11$-layer of reacting powder.

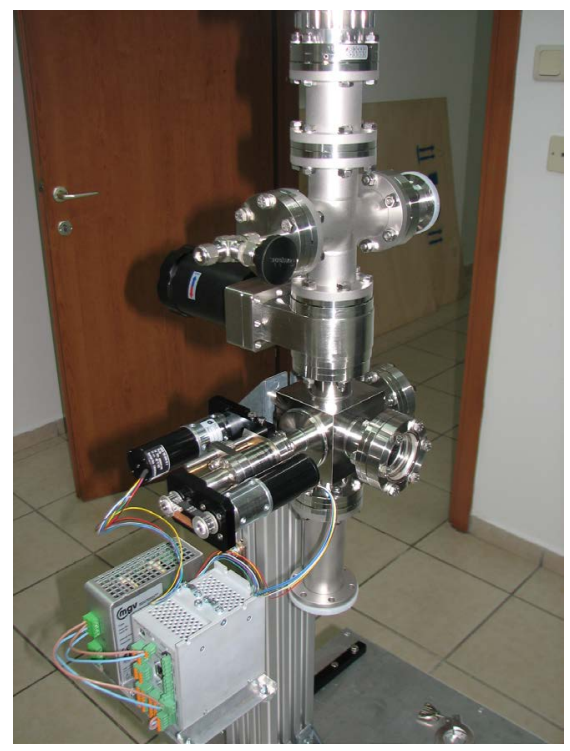

Figure 3. Laboratory scale mechanochemical sorption apparatus.

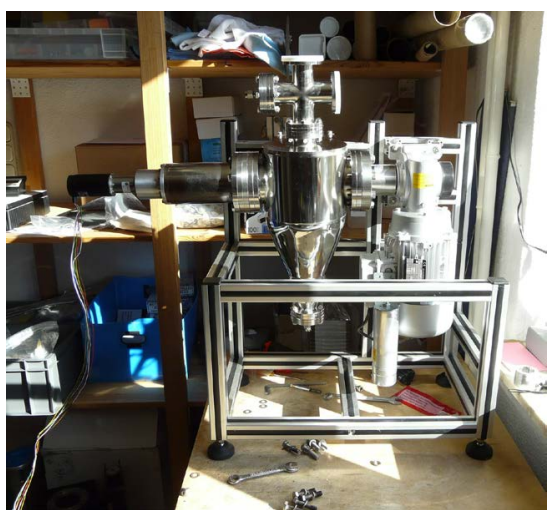

Figure 4. Pilot prototype of mechanochemical apparatus. 


\subsection{Maximal Product Yield}

Unlike the powder of the gas purifier as-milled particles in layer 11 (Figure 2) are saturated with defects of different kinds [17]. Such particles have high rate of impurity capturing, which is provided by surface and near surface layers with the thickness of up to several microns. In terms of the axial distribution $u(\xi, \tau)$ (Figure 1) this shows itself as a sharp step-like shape of the distribution. As a result the increase of impurity concentration in the purified outlet gas occurs much slower while coefficient $\eta$ of the reactant using increases up to $100 \mathrm{wt} \%$ if the typical size (i.e. the radius of a spherical or a needle-like particle, or the half-thickness of a plate-like particle) of the powder is the value in the range 10 15 micron.

\subsection{Control over Sorption Process}

The characteristics of the purification process can be governed by the control of the Me powder feed in the sorption column which was not available before. In particular, the purity of the gas product can be changed by adjusting the milling intensity to the gas flow rate. The respective gas purity analysis can be carried out via the sampling port 7 in Figure 2.

The difference between traditional and mechanochemical purifying apparatus is demonstrated by time dependent plots in Figure 5.

The curve $u_{p}(\tau) \equiv u(1, \tau)$ describes changes of gas purity in the outlet of a tubular purifier with the fixed sorption bed. The similar dependence for the mechanochemical apparatus $u_{m}(\tau)$ is obtained in conditions where the capturing rate is adjusted to the rate with which the impurity enters the apparatus. The projection onto axis $\tau$ of the intersection of curves $u_{p}(\tau)$ and $u_{m}(\tau)$ with line $u=u_{c}$ determines the moments of the termination of the operation $\tau_{p}$ or $\tau_{m}$ of the gas purification equipment. It is seen that the mechanochemical method of gas purification is maximally efficient, $\tau_{m} \gg \tau_{p}$, as the process stops practically with the exhaust of the ingot.

The above said means that the mechanochemical apparatus for the first time in real production allows approaching the limit of the sorption capacity of the purification material. To be more precise, under the limit we understand here the amount of gas $\mathrm{Y}$, which is captured by reactant $\mathrm{Me}$ in the reaction $\mathrm{Me}+\mathrm{Y}=\mathrm{MeY}$, which runs to completion.

One more feature of the purification process is related with the quality control of the outlet gas. In order to ensure gas purity in traditional fixed bed purifiers it is necessary to find time $\tau_{p}$ in advance (Figure 5) so that to be able to ensure either timely termination of the process or a switch to a bypass line with freshly charged purifier. This problem does not exist with the mechanochemical equipment because time $\tau_{m}$ (Figure 5) practically corresponds to the moment when the ingot is nearly entirely consumed in the grinding process. The respective reduction in the length of Me ingot can be easily tracked with the help of an outside position gauge.

The using of mechanochemical apparatus is quite wide. It can be utilized in 


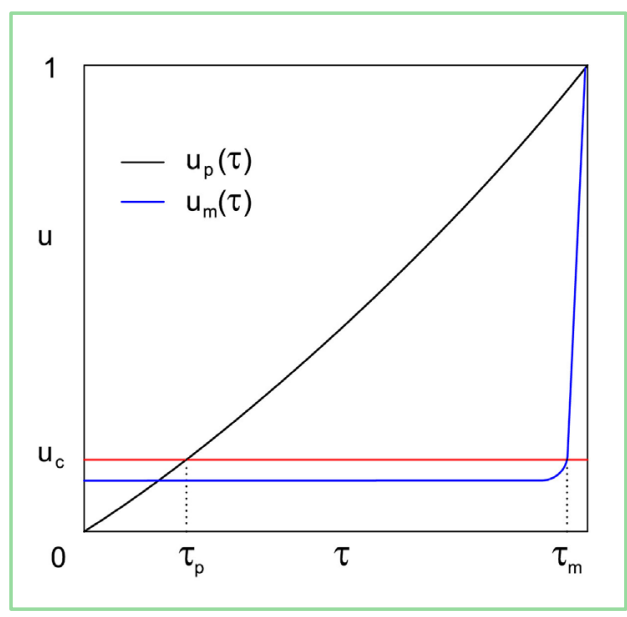

Figure 5. The dependence of the dimensionless impurity concentration in the outlet gas on the process time; $u_{p}(\tau)$-impurity concentration in the outlet of the gas purifier, $u_{m}(\tau)$-the impurity concentration at the outlet of the mechanochemical apparatus, $\tau_{p}$ and $\tau_{m}$-the working time of the gas purifier and the mechanochemical apparatuses correspondingly, $u_{c}$-see $u_{c}$ in Figure 1.

the production of high purity and ultra-pure gases where it surpasses traditional sorption units in many respects. The applications include vacuum mills for disintegration of highly reactive materials, the removal of residual gases such as oxygen, nitrogen, etc. from evacuated vessels or scientific tools in studies of gas-metal systems.

\section{Reactive Sorber}

In difference with mechanochemical apparatus where intensive grinding is used reactive sorbers utilize relatively weak surface scrubbing of the purification material. In a sense, such sorbers can be considered as hybridization of a gas purifier with a powder or granular reactant and a high pressure reactor with a magnetically stirred high-pressure reactor, Figure 6. Gas purification in the sorber takes place in the following way. Gas with impurity $\mathrm{Y}$ is fed from above into reactor II with particles 5 of reactant Me, which are stirred at a small speed by the blades of stirrer 7 . The formed on the surface Me products of the reaction of the composition MeY fall down in the process of mutual rubbing of the particles and are taken away by the gas flow to the waste collector III. The purified gas leaves along the gas line, which is located below the dividing mesh 8 and the products of reaction 12 in a form of nano- and micro-particles are collected in vessel 13.

Like the mechanochemical apparatus the sorber radically changes the situation in the production of pure gases creating the conditions under which getter material has enough time in the process of purification to react with gases to 


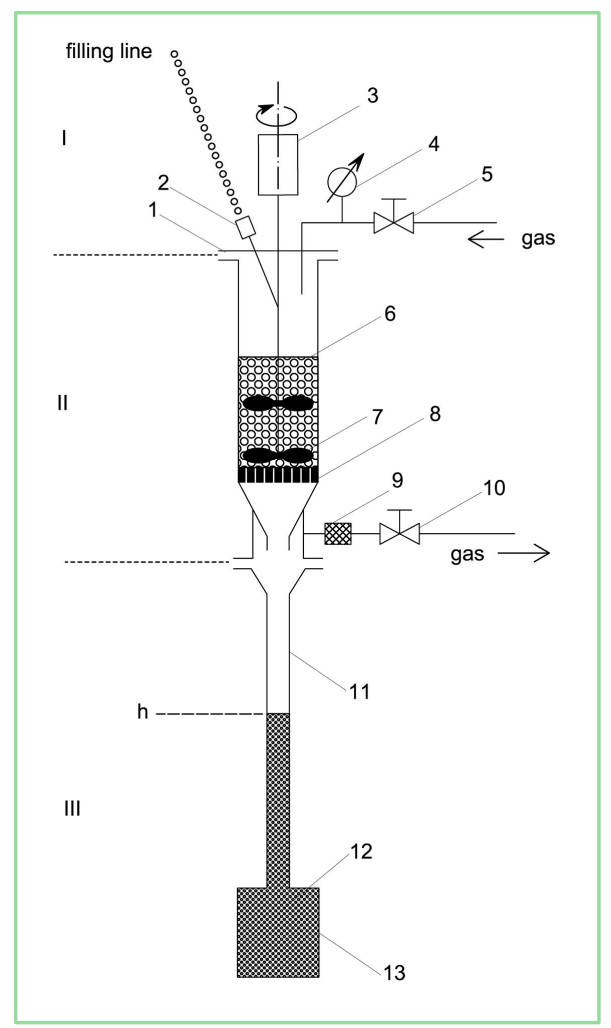

Figure 6. The sketch of reactive sorber. I-sorber head, II-high pressure reactor, III-bottom chamber; 1-flange, 2-charging port, 3-actuator of the magnetic stirrer, 4-manometer, 5-valve, 6-reactant, 7stirrer blades, 8-dividing mesh, 9-filter, 10-valve, 11-connecting tube, 12-waste, 13-waste collector; $h$ is the height of the accumulated waste.

completion. In fact, nano layers $\mathrm{MeY}$, which appear on the surface Me in the presence of $\mathrm{Y}$, are fragile, mechanically unstable and do not sit tightly on the boundary with Me. Already at the small stirring speed they are easily removed from the Me surface baring it and by this maintaining the kinetics of the reaction at the highest level unlike the processes taking place in gas purifiers, where reactions of gases with Me particles are limited by diffusive transfer through the layer of products. By changing the rotation speed of the stirrer it is possible to vary the ratio of areas $S_{\mathrm{MeY}} /\left(S_{\mathrm{Me}}+S_{\mathrm{MeY}}\right)$ from zero to one adjusting for the concrete needs. Here $S_{\mathrm{MeY}}$ is the surface area of the reactant covered with the product $\mathrm{MeY}$ and $S_{\mathrm{Me}}$ is the surface area of purely metallic regions.

The operation of the sorber in the case when $S_{\mathrm{MeY}} /\left(S_{\mathrm{Me}}+S_{\mathrm{MeY}}\right) \rightarrow 0$ during the entire process is schematically shown in Figure 7. In the given regime the specific sorption rate, i.e. the rate per unit of the mass of reactant $\mathrm{Me}$, is constant and close to that maximal value, which is observed for the fresh metallic surface. Nevertheless, the total rate of capturing gases decreases with time for the reason 


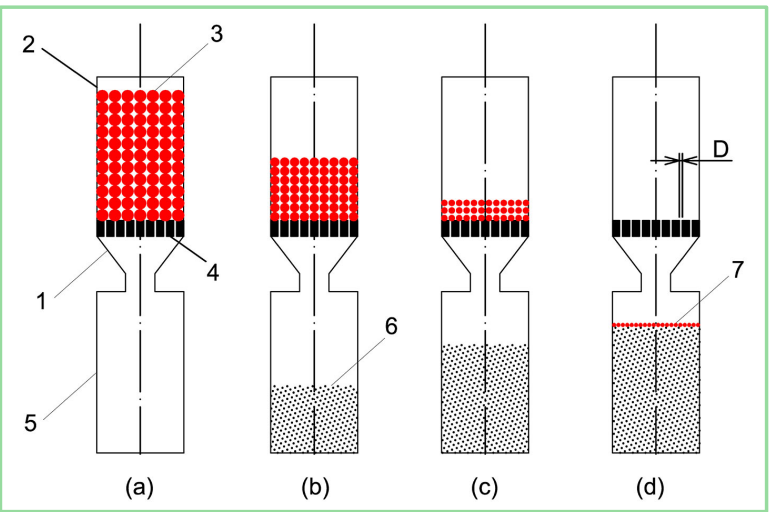

Figure 7. Schematic presentation of changes in the charge of sorption reactor with time. (a) - start, (b) and (c) - -intermediate stages, (d) - final stage; 1 -sorption column, 2-reactor, 3-particles of reactant $\mathrm{Me}$, 4-dividing mesh, 5-waste collector, 6-MeY particles, 7-residual Me particles with the size of smaller than the separator mesh.

of the "sorption wear" of the particles, particularly due to the decrease in their size and the decrease of the total surface area of reactant Me at the mechanical rubbing of the particles in gas medium.

This is demonstrated in Figure 7, where positions (a) and (d) answer the initial and final stages of the process correspondingly, and positions (b) and (c) - the intermediate ones. It is seen that the diameter of initial particles 3 gradually decreases and the amount of waste 6 grows. The purification process continues till the diameter of the particles is larger than the size of the openings $D$ of the dividing mesh 4 but stops as soon as the particles fall into vessel 5 reaching the value $D$ (Figure $7(\mathrm{~d})$ ).

\subsection{Production Yield}

The dividing mesh 8 (Figure 6) is an important tool for the efficiency increase and quality control of the gas purification process. As concerns the process efficiency, it is not difficult to show that the value of the coefficient $\eta$ is determined by the ratio $D_{r} / D$, where $D_{r}$ is the average value of the initial diameter of the Me particles. In fact, as it was said before, in the process of rubbing of the particles their consumable outside layers turn into nano- and micro-particles of the composition $\mathrm{MeY}$ and the diameter of the metal residual particles decreases until it becomes equal to the value $D$. By this moment the share of the reacted with gas reactant is $\eta=1-\left(D / D_{r}\right)^{3}$, where from it is seen how easily and convenient it is to influence the efficiency of the gas purification process via the values $D_{r}$ and $D$. To provide the freedom of manoeuvre the dividing meshes should be replaceable, which will allow adjusting the value $D_{r} / D$ to the desirable value both due to the initial size of the particles and with the help of the set of meshes having the openings of different diameter $D$.

Let's consider as an example the case where $D_{r} / D=5$ and, respectively, $\eta=$ 0.992. This means that more than $99 \%$ of the sorption material is used for puri- 
fication, and the only condition for such an advantageous result is the appropriate rotation of the stirrer that ensures formation of Me particles with $D_{r}$ five times larger than $D$. For instance, the mesh size must be $1 \mathrm{~mm}$ when $D_{r}=5 \mathrm{~mm}$, the mesh size must be $0.5 \mathrm{~mm}$ when $D_{r}=2.5 \mathrm{~mm}$, the mesh size must be $0.2 \mathrm{~mm}$, when $D_{r}=1 \mathrm{~mm}$, the mesh size must be $0.1 \mathrm{~mm}$ when $D_{r}=0.5 \mathrm{~mm}$, etc. So, it can be seen that there are no difficulties with the production of a mesh or the reactant for the sorber.

Depending on the application there can be different requirements to the sorber. However, like with any periodically charged sorption column the kinetics of the reactive sorption tends to decrease with time [6] because of the exhaustion of the purifying material. Respectively, the outlet gas may cease to meet the purity requirements. Hence, the purity of the outlet gas is the subject of continuous monitoring.

\subsection{Quality Control}

The separation of active $\mathrm{Me}$ and waste $\mathrm{MeY}$ particles with the dividing mesh 8 suggests the process control by measuring the waste height $h$ in the tubular-like part 11 of the collector in Figure 6. The rate of impurity capturing $j(t)$ is presented in Figure 8. The shaded area shows the amount $q\left(t^{*}\right)=\int_{0}^{t^{*}} j(t) \mathrm{d} t$ of sorbed gas at time $t^{*}$, which can be interrelated with formation of the waste mass $m_{\mathrm{MeY}}\left(t^{*}\right)=\left(1+M_{\mathrm{Me}} / M_{\mathrm{Y}}\right) q\left(t^{*}\right)$, where $M_{\mathrm{Me}}$ and $M_{\mathrm{Y}}$ stand for the atomic mass of the reactant and the impurity, respectively. The smallest allowed (critical) sorption rate $j_{c}$ at time $t_{c}$ occurs when the waste mass achieved $m_{\mathrm{MeY}}\left(t_{c}\right)=\left(1+M_{\mathrm{MeY}} / M_{\mathrm{Y}}\right) \int_{0}^{t_{c}} j(t) \mathrm{d} t$. Therefore, when the dependence $j=j(t)$ is known and the threshold $j_{c}$ for the gas capturing rate is set one can calculate the amount of waste $m_{\mathrm{MeY}}\left(t_{c}\right)$. This amount is equivalent to the waste level $h_{c}$ in tube 11 (Figure 6). As a result, the tubular part of the waste collector can be appropriately scored in order to serve as a gauge for the process control and termination.

Another way of solving the problem of economical safety of the process systems using gas from the reactive sorber is possible when the sorber is combined with the purity indicator created on the basis of the gas flow meter [9]. The readings of this indicator are labeled in the units of concentration of the target gas according to the results of the calibration tests, which apart from the gas flow meter involve also the sorber and the precision analytical equipment. As a result the sorber and the mentioned indicator form together a highly efficient and controllable gas purification system, which is capable of stabilization the rate of capturing gases at the set level by way of adjusting the rotation rate of the stirrer according to the readings of the purity indicator.

Reactive sorbers can considerably improve operation and economic performance of gas purification. Their efficiency can be several orders of magnitude higher while purification costs per unit volume much smaller compared to traditional sorption apparatus. Therefore, they can be applied even for purification of 


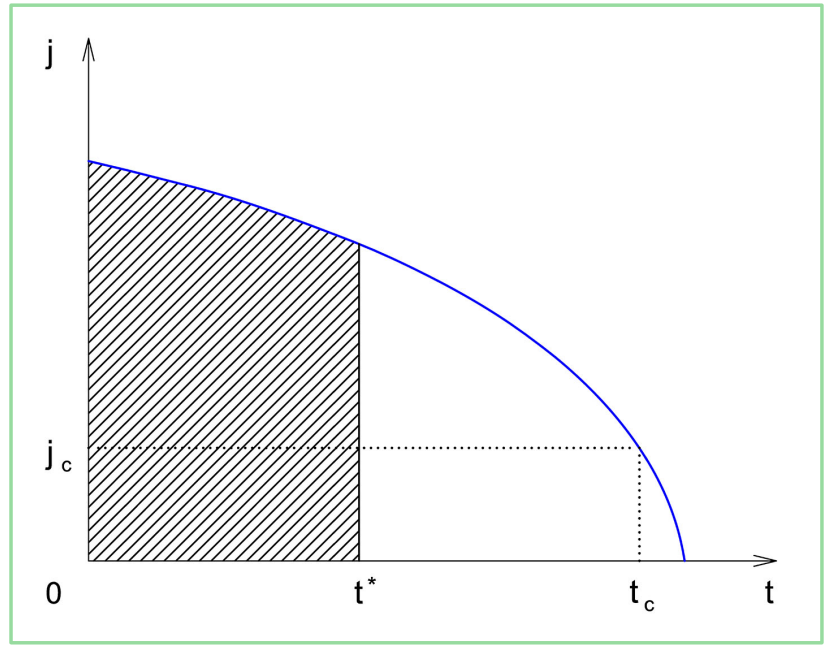

Figure 8. Gas capturing rate $j$ in the reactive sorber as a function of time $t$. $j_{c}$-critical rate below which the gas quality becomes unsatisfactory, $t^{*}$-running time, $t_{c}$-time of safe operation of the sorber.

gases with rather large concentration of impurities, i.e. in conditions where the using of traditional fixed bed sorbers is either very costly or just impossible. Examples include recycling of helium and other noble gases that may contain up to $10 \%-20 \%$ of oxygen, nitrogen and other admixtures, the obtaining of nitrogen directly from air and maintaining nitrogen atmosphere in glove boxes and storage cabinets, one-step hydrogen purification, etc.

\section{Consumables}

Reactants continuously interact with gas impurities in normal conditions according to the parabolic or linear law [6]. For capturing any gas impurity except noble gases it is practically always possible to select the metal, which in a mechanically activated state is capable of entering into a chemical reaction with this impurity, forming a nonvolatile chemical compound. First of all, these are alkali and alkali-earth metals and some of the rare-earth metals.

Besides the chemical affinity to active and low active gases reactants possess one more necessary feature. Namely, in binary, ternary and more multicomponent alloys with each other they form such phase mixtures or phases as eutectics and low melting intermetallic compounds, which are at the same time fragile and mechanically weak while the chemical compounds with gases, which are formed on their surface, are easily removed with small efforts. Although these facts have been known for many years only now they find practical application. Alloying of $\mathrm{Ca}, \mathrm{Li}$, and $\mathrm{Mg}$ occurs sufficient for most applications while other reactive metals are sometimes can be used as small additives. This is the optimal variant, which takes into consideration the working properties of reactants, the technology of their production, costs and availability. Generally, the production cost of such reactants is much smaller compared to non evaporable getters 
(NEGs) where transition metals like $\mathrm{Ti}, \mathrm{V}, \mathrm{Zr}$ and others are used. Without going into details let's only mention some facts to support this assertion.

The standard sintered porous bodies used in NEGs are the products of a 4 - 5 stage production process while reactants can be manufactured in one or two stages. The melting temperatures of the transition metals are 4 - 5 times higher than the temperatures necessary for melting alkali and alkali-earth alloys and, consequently, it makes much simpler the manufacturing of ingots for milling in mechanochemical apparatus or cast granules of reactants for sorbers. Moreover, the price of starting materials for alloying is considerably higher for transition metals than for alkali and alkali-earth metals. It is important to mention also abundance, nontoxicity of $\mathrm{Ca}, \mathrm{Mg}$ and $\mathrm{Li}$, and well-mastered, reliable and low-cost production methods of these metals from the natural raw materials or from industrial wastes.

Here an unavoidable question arises, how it happened that although advantages of powder reactants are clear they have not taken the appropriate place in sorption technologies. The answer can be received from the analysis of the attempt of gettering the residual gases in vacuum vessels by $\mathrm{Ba}$ alloys [18] [19]. According to these documents the initial ingots were ground in a glove box under Ar and then the products of grinding were transferred into a vacuum vessel. The way it was done was not disclosed and according to the data of the sorption measurements [18] [19] the consumption of the reactive alloy did not exceed $2 \%$ of its total mass. This is an ordinary result and besides, the problem with the waste containing about $98 \%$ of reactive metal appeared. So, remaining within the frames of the standard technology of transferring the sorbent from the place of its production into the sorption column it is not possible to implement the entire sorption potential even in the case of reactive metals. Entirely new steps for activating metallic chemisorbents were necessary, which used ideas and practical techniques mastered in the field of mechanochemistry.

The new gas purification equipment opens up wide prospects for sorption technologies due to increased quality of gas products at lower price and improved service level as well as the appearance of new products. The reactive sorption equipment is the result of the application of mechanochemical methods to metallic chemisorbents. Such a combination leads to a great progress in economics and production of pure gases. Estimates show that purification costs can be more than 10000 times smaller compared with the current methods.

\section{Conclusions}

1) Mechanochemical methods in their application to reactants become the governing force in the development of sorption technologies. It is these methods that allow the complete realization of the sorption potential of reactants in the ambient temperature, i.e. without heating or cooling.

2) The implementation of mechanochemical activation depends on the sorption problem. One variant of the activation is mechanical destruction of the solid 
with saturation of its fragments with a big amount of defects. As a result not only the surface but also the volume of the material is involved in reactions with gases.

3) Another variant of mechanochemical activation is the surface rubbing, which allows maintaining the sorption at the maximally high level due to the continuous renewal of the reacting surface.

4) The independent factor that can be used for governing the sorption process is related with mechanical work performed by milling cutter or agitating stirrer. Controlling the work of these tools allows changing the time dependent form of the sorption curve in such a way that the value of the coefficient of using $\eta$ may approach unity.

5) The dividing mesh plays an important role in reactive sorption process. The coefficient of sorbent using depends on the mesh size, which can be selected in such a way as to ensure practically complete consumption of the reactant. The dividing mesh and waste collector are prerequisites of a simple design for quality control of the outlet gas.

\section{Acknowledgements}

V.L. Kozhevnikov is grateful for partial support of this work under government project №18-10-3-32.

\section{References}

[1] della Porta, P. (1992) "Gettering” an Integral Part of Vacuum Technology, Technical Paper TP 202. AVS 39th, National Symposium, 9-13 November 1992.

[2] Carrea, G. and Warrick, B.D. (1999) Low Temperature Inert Gas Purifier. US Patent 5902561 .

[3] Zeller, R. and Vroman, C. (2006) Porous Sintered Composite Materials. US Patent 7112237.

[4] Murphy, W., Edlund, E. and Brown. K. (2008) Purifier. US Patent 7438864.

[5] Chuntonov, K. and Setina. J. (2017) Activationless Gas Purifiers with High Sorption Capacity. US Patent 9586173.

[6] Chuntonov, K., Atlas, A., Setina, J. and Douglass, G. (2016) Getters: From Classification to Materials Design. Journal of Materials Science and Chemical Engineering, 4, 23-34.

[7] Chuntonov, K., Setina, J. and Douglass. G. (2015) The Newest Getter Technologies: Materials, Processes, Equipment. Journal of Materials Science and Chemical Engineering, 3, 57-67. https://doi.org/10.4236/msce.2015.39008

[8] Chuntonov, K. and Setina. J. (2016) Reactive Getters for MEMS Applications. Vacuum, 123, 42-48. https://doi.org/10.1016/j.vacuum.2015.10.012

[9] Chuntonov, K., Ivanov, A.O., Verbitsky, B. and Kozhevnikov, V.L. (2017) Gas Purification and Quality Control of the End Gas Product. Journal of Materials Science and Chemical Engineering, 5, 44-58.

[10] Alvarez Jr., D. (2001) Method and Apparatus for Purification of Hydride Gas Streams. US Patent 6241955.

[11] Weber, D.K. and Vergani, G. (2003) Rejuvenable Ambient Temperature Purifier. 
US Patent 6521192.

[12] Baker, J.D., Meikrantz, D.H., Pawelko, R.J., Anderl, R.A. and Tuggle, D.G. (1994) Tritium Purification via Zirconium-Manganese-Iron Alloy Getter St 909 in Flow Processes. Journal of Vacuum Science \& Technology A, 12, 548. https://doi.org/10.1116/1.579167

[13] Succi, M. and Solcia, C. (2002) Hydrogen Purification. US Patent 6436352.

[14] Brochure SAES Getters. MonoTorr, PS4 Series, 2003.

[15] Chuntonov, K. and Lee. M.K. (2014) Mechanochemical Sorption Apparatuses. Advanced Material Research, 875-877, 1106-1110. https://doi.org/10.4028/www.scientific.net/AMR.875-877.1106

[16] Chuntonov, K. (2015) Sorption Apparatuses for the Production of Pure Gases. US Patent 9095805

[17] Meyer, K. (1968) Physikalisch-Chemische Kristallographie. VEB Deutscher Verlag fur Grundstoffindustrie, Leipzig.

[18] Boffito, C. and Schiabel, A. (1994) Process for the Sorption of Residual Gas by Means of a Non-Evaporated Barium Getter Alloy. US Patent 5312606.

[19] Schiabel, A. and Boffito, C. (1994) Process for the Sorption of Residual Gas by Means by a Non-Evaporated Barium Getter Alloy. US Patent 5312607. 